\title{
Intervenções de enfermagem para prevenção de dermatite periestoma em estomas
}

\section{intestinais: Revisão sistemática}

\author{
Nursing interventions for the prevention of peristomal dermatitis in intestinal stomas: A systematic \\ review
}

\section{Intervenciones de enfermería para la prevención de la dermatitis peristómica en estomas}

intestinales: Revisión sistemática

Recebido: 02/06/2021 | Revisado: 13/06/2021 | Aceito: 15/06/2021 | Publicado: 30/06/2021

Sindy Lamônie do Espírito Santo Barbosa

ORCID: https://orcid.org/0000-0003-3811-3089 Universidade Federal de Sergipe, Brasil

E-mail: contato.sindylamonie@gmail.com

Fernanda Oliveira de Carvalho

ORCID: https://orcid.org/0000-0002-1270-406X Universidade Federal de Sergipe, Brasil E-mail: fsoliveira.fisio@gmail.com

Isla Evellen Santos Souza

ORCID: https://orcid.org/0000-0002-0703-7493 Universidade Federal de Sergipe, Brasil E-mail: ievellenss@gmail.com

Lidiane Souza Lima

ORCID: https://orcid.org/0000-0002-3615-2159 Universidade Federal de Sergipe, Brasil. E-mail: lidi_lima88@hotmail.com

Neylor Rodrigo Oliveira Aragão ORCID: https://orcid.org/0000-0002-5283-511X Universidade Federal de Sergipe, Brasil E-mail: wilde_br@yahoo.com.br

Caíque Jordan Nunes Ribeiro

ORCID: https://orcid.org/0000-0001-9767-3938 Universidade Federal de Sergipe, Brasil

E-mail: caiquejordan_enf@yahoo.com.br

Vera Lúcia Conceição de Gouveia Santos ORCID: https://orcid.org/0000-0002-1288-5761

Universidade de São Paulo, Brasil E-mail: veras@usp.br

Ana Cristina Freire Abud

ORCID: https://orcid.org/0000-0002-3314-2182 Universidade Federal de Sergipe, Brasil E-mail: acfabud@uol.com.br

\begin{abstract}
Resumo
Introdução: Apesar dos avanços nas técnicas cirúrgicas de confecção e nos cuidados às pessoas com estomas, as dermatites periestomas de estomas intestinais são frequentes. Nesse contexto, a enfermagem assume papel fundamental para a prevenção dessas complicações. Objetivo: Realizar uma revisão sistemática para identificar intervenções de enfermagem utilizadas como medidas de prevenção de dermatite periestoma em estomias intestinais. Método: Realizou-se uma revisão sistemática nas bases de dados Scopus, PubMed (MEDLINE), Cochrane Library, Web of Science e CINAHL. Foram selecionados ensaios controlados randomizados que investigassem a eficácia das intervenções de enfermagem na prevenção de dermatite periestoma, publicados nos idiomas inglês, português e espanhol, até dezembro de 2020. Resultados: Inicialmente, 2.976 estudos foram identificados, no entanto apenas três desses foram incluídos na amostra final, os quais foram publicados entre 2011 e 2017. As ações de prevenção encontradas nos ensaios clínicos versaram sobre medidas educativas, uso de barreira cutânea moldável e padronização da técnica de limpeza e uso de pó de hidrocoloide como prevenção. Conclusão: As evidências sobre as intervenções de enfermagem no cuidado preventivo de lesões periestomas ainda são escassas e com riscos de viés consideráveis. Dessa maneira, encoraja-se a comunidade científica a realizar estudos primários de alto rigor metodológico, a fim de subsidiar a prática da estomaterapia e promover uma assistência de qualidade e baseada nas melhores evidências científicas.
\end{abstract}

Palavras-chave: Cuidados de enfermagem; Dermatite; Estoma; Prevenção; Revisão sistemática. 


\begin{abstract}
Introduction: Despite advances in surgical techniques for making and caring for people with stomas, peristomal dermatitis of intestinal stomata is frequent. In this context, nursing plays a fundamental role in preventing these complications. Objective: To carry out a systematic review to identify nursing interventions used as preventive measures for peristomal dermatitis in intestinal ostomies. Method: A systematic review was carried out in the Scopus, PubMed (MEDLINE), Cochrane Library, Web of Science and CINAHL databases. Randomized controlled trials investigating the effectiveness of nursing interventions in preventing peristomal dermatitis, published in English, Portuguese and Spanish until December 2020, were selected. Results: Initially, 2,976 studies were identified, however only three of these were included in the final sample, which were published between 2011 and 2017. Prevention actions found in clinical trials were about educational measures, use of moldable skin barrier and standardization of cleaning technique and use of hydrocolloid powder as prevention. Conclusion: Evidence on nursing interventions in the preventive care of peristomal injuries is still scarce and with considerable risk of bias. Thus, the scientific community is encouraged to carry out primary studies of high methodological rigor, in order to support the practice of stomatherapy and promote quality care based on the best scientific evidence.
\end{abstract}

Keywords: Nursing care; Dermatitis; Stoma; Prevention; Systematic review.

\title{
Resumen
}

Introducción: A pesar de los avances en las técnicas quirúrgicas para la confección y el cuidado de personas con estomas, la dermatitis periestomal de los estomas intestinales es frecuente. En este contexto, la enfermería juega un papel fundamental en la prevención de estas complicaciones. Objetivo: Realizar una revisión sistemática para identificar las intervenciones de enfermeira utilizadas como medidas preventivas de la dermatitis periestomal en las ostomías intestinales. Método: Se realizó una revisión sistemática en las bases de datos Scopus, PubMed (MEDLINE), Cochrane Library, Web of Science y CINAHL. Se seleccionaron ensayos controlados aleatorizados que investigan la efectividad de las intervenciones de enfermería en la prevención de la dermatitis periestomal, publicados en inglés, portugués y español hasta diciembre de 2020. Resultados: Inicialmente, se identificaron 2.975 estudios, sin embargo, solo tres de estos se incluyeron en la muestra final, que se publicaron entre 2011 y 2017. Las acciones de prevención encontradas en los ensayos clínicos fueron sobre medidas educativas, uso de barrera cutánea moldeable y estandarización de la técnica de limpieza y uso de hidrocoloide en polvo como prevención. Conclusión: La evidencia sobre las intervenciones de enfermería en la atención preventiva de las lesiones periestomales es aún escasa y con considerable riesgo de sesgo. Así, se incentiva a la comunidad científica a realizar estudios primarios de alto rigor metodológico, con el fin de apoyar la práctica de la estomaterapia y promover una atención de calidad basada en la mejor evidencia científica.

Palabras clave: Atención de enfermería; Dermatitis; Estoma; Prevención; Revisión sistemática.

\section{Introdução}

A manutenção da integridade da pele é um dos principais objetivos da assistência em enfermagem a pacientes com estomias. No entanto, apesar dos avanços nas técnicas cirúrgicas de construção de estomas e melhoria dos cuidados às pessoas com essa condição, as complicações imediatas, mediatas e tardias da pele periestoma ainda são bastante frequentes (Carlsson et al., 2016).

A incidência de lesões de pele periestoma é difícil de ser determinada devido à heterogeneidade metodológica dos estudos. Acredita-se que exista um risco variável de 30 a 67\% de ocorrência de complicações na pele periestoma, sendo mais frequente a dermatite química ou irritativa, na qual o dano cutâneo ocorre quando a pele é exposta a efluentes do estoma, resultando em inflamação e erosão (Almutairi, LeBlanc \& Alavi, 2017; Gray et al., 2013; Nadal, Peñalba \& Zamora, 2007; Salvadalena, 2007). O desenvolvimento dessas alterações pode levar os pacientes a terem até três vezes mais chances de readmissão hospitalar além de provocar impacto altamente danoso no processo de reabilitação (Wick et al., 2011).

Os efeitos danosos da umidade sobre a pele (moisture associated skin damage - MASD) consistem em lesões e/ou processos inflamatórios resultantes da interação entre fatores mecânicos, microbianos ou químicos (fezes, urina, suor, exsudatos ou saliva), comorbidades, extremos de idade e uso de agentes irritantes para higienização. Qualquer um desses fatores pode vir ocasionar um desequilíbrio homeostático da pele ou qualquer tipo de dermatite, que impactam diretamente a reabilitação, a qualidade de vida e o bem-estar do paciente (Gray et al., 2011).

Portanto, além dos desafios físicos decorrentes do processo cirúrgico e da adaptação, as pessoas com uma estomia precisam de estratégias de educação em saúde e na utilização de técnicas e recursos disponíveis voltados para o autocuidado, 
prevenção e recuperação de complicações, visando a uma assistência segura que resulte em melhores desfechos para essa população (Silva et al., 2019).

$\mathrm{O}$ conhecimento adequado das intervenções de enfermagem baseadas em evidências pode fundamentar medidas preventivas direcionadas à manutenção da integridade da pele periestoma das pessoas com estomias. A prevenção tem impacto direto sobre a economia dos serviços, ao gerar menores custos diretos e indiretos quando comparados ao tratamento e à reabilitação (Loro et al., 2016). É bastante preocupante que depois de tantos anos da implantação de uma especialidade internacional como a Estomaterapia, a literatura ainda aponte taxas tão elevadas de uma complicação prevenível como a dermatite periestoma. Dessa forma, o objetivo desse estudo foi realizar uma revisão sistemática para identificar intervenções de enfermagem utilizadas como medidas de prevenção de dermatite periestoma em estomias intestinais.

\section{Metodologia}

Tipo de estudo

Trata-se de uma revisão sistemática que seguiu as recomendações preconizadas pelo Assessment of Multiple Systematic Reviews (AMSTAR) (Shea et al., 2007) e pelo Preferred Reporting Items for Systematic Reviews and MetaAnalyses (PRISMA) (Moher, Liberati, Tetzlaff \& Altman, 2009), cujo protocolo foi registrado no International Prospective Register of Systematic Reviews (PROSPERO - CRD42020179470). As revisões sistemáticas são estudos secundários que identificam um conjunto de evidências provenientes de estudos primários para responder a uma questão de pesquisa bem delimitada. Além disso, por meio de uma avaliação cuidadosa dos estudos incluídos, permite concluir a respeito da qualidade global das evidências existentes, auxiliando os clínicos e aos tomadores de decisão sobre as melhores práticas em saúde (Hulley et al., 2013).

\section{Questão de pesquisa e critérios de elegibilidade}

Este estudo foi desenvolvido para responder seguinte questão: "Quais são as intervenções de enfermagem empregadas na prevenção de dermatite periestoma de estomas intestinais?”. Utilizou-se a estratégia PICOT para direcionar a questão clínica, os critérios de elegibilidade e as estratégias de busca: $\mathrm{P}$ (população): pacientes com estomas intestinais; I (intervenção): medidas preventivas empregadas pela equipe de enfermagem; C (controle): cuidado usual; O (desfecho): prevenção de dermatite periestoma; e T (tipo de estudo): ensaios controlados randomizados (ECRs).

Foram incluídos ECRs que investigassem a eficácia de cuidados de enfermagem para a prevenção para dermatites periestoma, em pacientes com estomas intestinais, que apresentassem texto completo disponível, publicados até dezembro de 2020, nos idiomas espanhol, português e inglês. Excluíram-se estudos e séries de casos, editoriais, cartas ao editor, comentários, relatos de experiência, revisões (narrativas, integrativas, de escopo e sistemáticas), estudos observacionais (estudos transversais, caso-controle, coortes prospectivas ou retrospectivas, ou aninhados) e estudos experimentais que avaliaram intervenções para cicatrização de lesões periestoma ou preventivas empregadas por outros profissionais.

\section{Estratégia de busca}

Os descritores foram selecionados a partir dos Descritores em Ciências da Saúde (DeCS) e do Medical Subject Headings (MESH). As buscas foram realizadas, entre os meses de janeiro e fevereiro de 2021, por duas revisoras de maneira independente nas seguintes bases de dados: PubMed (MEDLINE), Cochrane Library, Web of Science e Cumulative Index to Nursing and Allied Health Literature (CINAHL). Em relação aos descritores, a busca foi guiada por diferentes combinações: "ostomy", "colostomy", "ileostomy", "dermatitis", "irritant dermatites", "skin manifestations", "nursing care”, "skin care", "prevention". Os descritores foram articulados com operadores booleanos "AND” e "OR" para a construção das estratégias 
entre as diferentes bases de dados. Além disso, foi realizada busca manual nas listas de referências dos estudos selecionados para análise final.

\section{Seleção dos estudos}

Os dados obtidos foram exportados para uma planilha eletrônica e as duplicatas removidas manualmente. Inicialmente, duas revisoras de forma independente triaram os estudos por meio da leitura dos títulos e resumos. Posteriormente, foi realizada a leitura na íntegra dos estudos triados para aplicação dos critérios de elegibilidade e exclusão. Em ambas as etapas, os estudos foram selecionados por meio de consenso entre as investigadoras. Quando não houve consenso, um terceiro revisor foi consultado. A concordância entre as revisoras foi mensurada por meio do teste estatístico Kappa, considerando a seguinte classificação: baixa concordância $(\kappa<0,40)$, boa concordância $(0,40<\kappa<0,75)$ e excelente concordância $(\kappa>0,75)$ (Hosmer \& Lameshow, 1989).

\section{Extração, síntese e análise qualitativa dos dados}

Os dados foram extraídos em planilha eletrônica padronizada, elaborada pelos autores, contendo as seguintes informações: autor, país, ano, tipo de estudo, amostra, critérios de inclusão, follow-up, intervenção, avaliação do desfecho e resultados. A análise qualitativa dos dados foi organizada em categorias temáticas. Devido à heterogeneidade dos estudos incluídos, não foi possível realizar a síntese quantitativa (metanálise) dos resultados.

\section{Análise do risco de viés}

O risco de viés dos artigos incluídos na amostra final também foi avaliado de forma independente por duas revisoras e os desacordos resolvidos por consenso. A ferramenta da Colaboração Cochrane para avaliação de ECRs foi utilizada para avaliar o risco de viés em baixo, incerto ou alto (Carvalho, Silva \& Grande, 2013). Esse instrumento avalia os riscos de vieses em sete domínios, sejam eles: geração de sequência e distribuição de alocação (viés de seleção), cegamento de participantes e pessoal (viés de desempenho), cegamento de avaliação de resultados (viés de deteç̧ão), dados de resultados incompletos (viés de atrito), relatos de resultados seletivos (viés de relato) e outras fontes potenciais de viés.

\section{Resultados}

O fluxograma da Figura 1 resume o processo de seleção dos artigos para a revisão sistemática e segue a metodologia do PRISMA. 
Figura 1. Fluxograma PRISMA de seleção dos estudos.
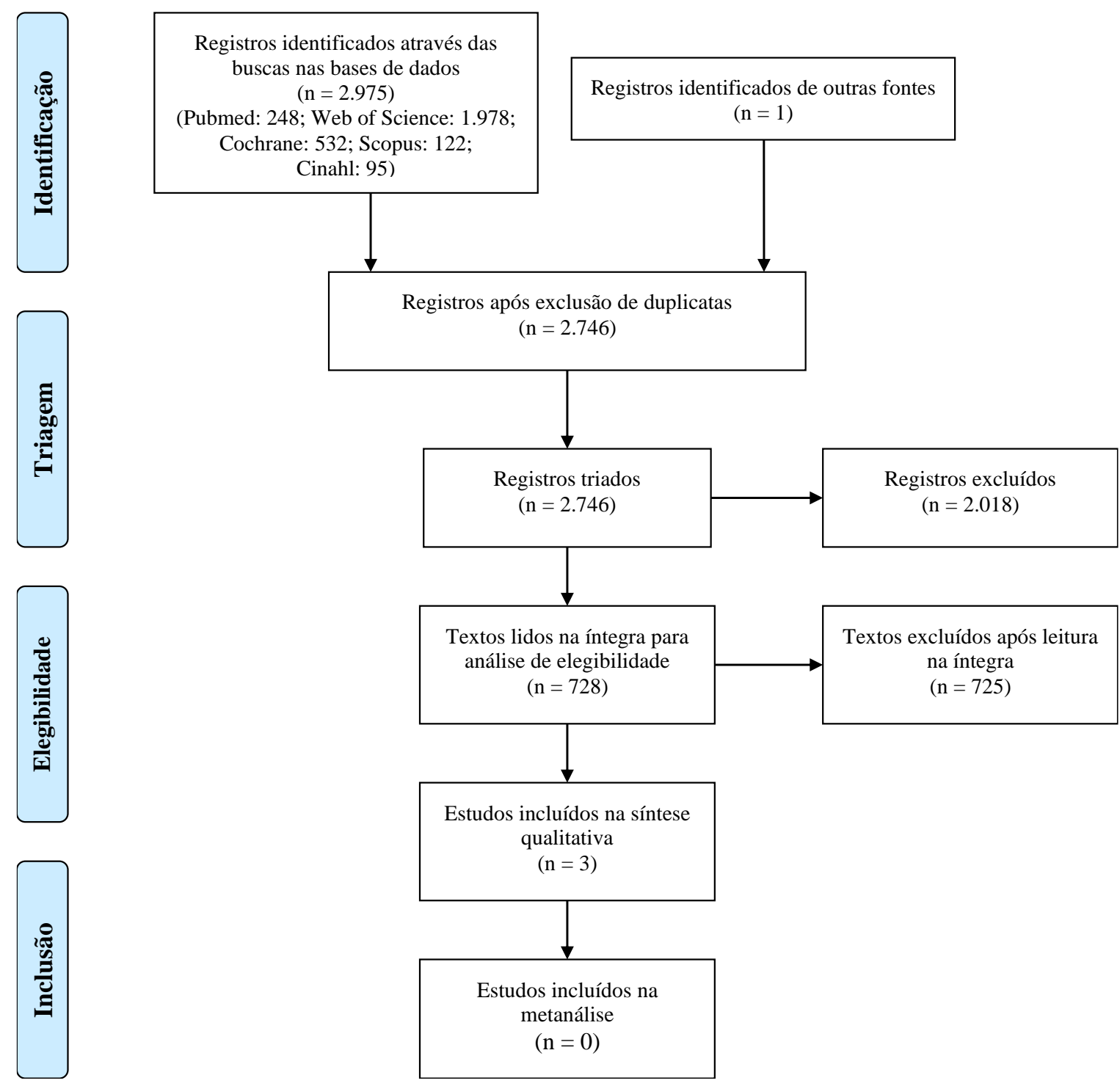

Fonte: Autores.

Um total de 2.976 artigos retornaram da busca, dos quais 230 foram excluídos por estarem duplicados e 2.018 não possuíam título e resumo relevantes para o estudo. Dos 728 artigos selecionados para a leitura na íntegra, apenas três atenderam aos critérios de elegibilidade e foram incluídos na análise final. Não foram incluídos trabalhos adicionais no estudo após análise de todas as listas de referências dos artigos selecionados por meio da busca manual. Houve um alto nível de concordância entre os revisores durante todas as etapas de seleção dos estudos $(\kappa>0,75)$.

\section{Características dos estudos incluídos}

Os três ECRs selecionados foram publicados entre os anos de 2011 e 2017 e realizados em diferentes países do continente asiático, sendo eles, a Arábia Saudita (1), China (1) e Coreia (1). A Tabela 1 traz as principais características dos estudos selecionados. 
Tabela 1. Características dos estudos incluídos na análise final.

\begin{tabular}{|c|c|c|c|c|c|c|c|}
\hline $\begin{array}{c}\text { Autor, ano e } \\
\text { país }\end{array}$ & Título & $\begin{array}{l}\text { Amostra } \\
\text { (GE e GC) }\end{array}$ & Critérios de inclusão & Follow-up & Intervenção & Avaliação do desfecho & Principais achados \\
\hline $\begin{array}{l}\text { Alenezi e } \\
\text { Mansour, } 2016 . \\
\text { Arábia Saudita }\end{array}$ & $\begin{array}{l}\text { Impacto da educação } \\
\text { sobre o cuidado do } \\
\text { estoma na redução da } \\
\text { incidência de } \\
\text { complicações cutâneas } \\
\text { do estoma. }\end{array}$ & $\begin{array}{l}\mathrm{GE}=50 \\
\mathrm{GC}=50\end{array}$ & $\begin{array}{c}\text { Idade > } 18 \text { anos; Cirurgia } \\
\text { colorretal eletiva com } \\
\text { formação de um estoma. }\end{array}$ & $\begin{array}{l}1,3 \text { e } 6 \\
\text { semanas } \\
\text { pós-alta. }\end{array}$ & $\begin{array}{l}\text { Programa educacional } \\
\text { sobre cuidados com o } \\
\text { estoma }\end{array}$ & $\begin{array}{l}\text { Aplicação de pré e pós } \\
\text { teste e utilização do } \\
\text { instrumento SACS } \\
\text { para o follow-up. }\end{array}$ & $\begin{array}{l}\text { Diminuição significativa } \\
\text { nas complicações } \\
\text { cutâneas periestomais no } \\
\text { GE }(\mathrm{p}=0,028) .\end{array}$ \\
\hline $\begin{array}{l}\text { Liu et al., } 2017 . \\
\text { China }\end{array}$ & $\begin{array}{l}\text { A aplicação da barreira } \\
\text { cutânea moldável no } \\
\text { autocuidado de idosos } \\
\text { estomizados. }\end{array}$ & $\begin{array}{l}\mathrm{GE}=56 \\
\mathrm{GC}=48\end{array}$ & $\begin{array}{l}\text { Idade entre } 65 \text { a } 79 \text { anos, } \\
\text { confecção de colostomia } \\
\text { devido ao câncer } \\
\text { colorretal; concordância } \\
\text { na participação do } \\
\text { estudo; ausência de } \\
\text { problemas de saúde } \\
\text { mental e capacidade de } \\
\text { entender instruções, } \\
\text { utilizar o equipamento de } \\
\text { ostomia, } \\
\text { e substituir o } \\
\text { equipamento de ostomia. }\end{array}$ & $\begin{array}{l}\text { No } \\
\text { momento } \\
\text { zero e } 1 \\
\text { mês pós- } \\
\text { alta. }\end{array}$ & $\begin{array}{l}\text { Uso da barreira cutânea } \\
\text { moldável desde a } \\
\text { formação do estoma. } \\
\text { Limpeza prévia com } \\
\text { solução salina ou } \\
\text { água destilada. }\end{array}$ & $\begin{array}{l}\text { Medição da incidência de } \\
\text { dermatite perilesional e } \\
\text { dos níveis de satisfação e } \\
\text { autocuidado por meio de } \\
\text { um questionário. }\end{array}$ & $\begin{array}{c}\text { A incidência de } \\
\text { dermatite irritante } \\
\text { periestomal foi menor no } \\
\text { GE }(\mathrm{p}<0,05) \text {. A } \\
\text { pontuação total de } \\
\text { satisfação do paciente do } \\
\text { GE foi superior ao do } \\
\text { GC }(\mathrm{p}<0,05) .\end{array}$ \\
\hline $\begin{array}{l}\text { Park et al., } \\
2011 . \\
\text { Coreia }\end{array}$ & $\begin{array}{c}\text { Comparação de } \\
\text { cuidados com a pele } \\
\text { periestomais } \\
\text { padronizados e técnica } \\
\text { de formação de crostas } \\
\text { na prevenção de } \\
\text { problemas cutâneos } \\
\text { periestomais em } \\
\text { pacientes com ostomia. }\end{array}$ & $\begin{array}{l}\mathrm{GE}=45 \\
\mathrm{GC}=36\end{array}$ & $\begin{array}{l}\text { Idade entre } 20 \text { a } 80 \text { anos, } \\
\text { ausência de diabetes } \\
\text { mellitus, doença vascular } \\
\text { ou doença } \\
\text { cardiovascular, } \\
\text { compreensão dos } \\
\text { objetivos e concordância } \\
\text { em participar do estudo. }\end{array}$ & $\begin{array}{l}1,2 \text { e } 3 \\
\text { meses pós- } \\
\quad \text { alta. }\end{array}$ & $\begin{array}{l}\text { Cuidados padronizados } \\
\text { para a pele peristoma. } \\
\text { Limpeza com água e } \\
\text { aplicação direta de } \\
\text { equipamentos } \\
\text { coletores. Aplicação de } \\
\text { filme barreira nos casos } \\
\text { de pacientes com } \\
\text { urostomia, idosos e } \\
\text { fragilidade da pele. }\end{array}$ & $\begin{array}{l}\text { Utilização do } \\
\text { instrumento OST para o } \\
\text { follow-up. }\end{array}$ & $\begin{array}{l}\text { O status no GE teve uma } \\
\text { possibilidade } 57 \% \text { menor } \\
\text { do que no GC. }\end{array}$ \\
\hline
\end{tabular}

GC: grupo controle; GE: grupo experimental; OST: Ostomy Skin Tool; SACS ${ }^{\mathrm{TM}}$ : Studio Alterazoni Cutanee Stomale. Fonte: Autores. 
O tamanho das amostras variou de 45 a 56 indivíduos para o grupo experimental (GE) e de 36 a 50 , para o grupo controle (GC). Os estudos tiveram como participantes pacientes adultos e idosos que realizaram a confecção de um estoma intestinal (colostomia ou ileostomia). Durante a análise qualitativa dos resultados, emergiram três categorias temáticas: intervenção, seguimento e desfecho.

\section{Intervenção}

No ensaio de Alenezi e Mansour (2016), realizado na Arábia Saudita, avaliou-se a eficácia da utilização de medidas educativas sobre cuidados com o estoma em pacientes adultos que realizaram cirurgia colorretal eletiva, com a formação de um estoma. A amostra foi composta de 100 pacientes selecionados aleatoriamente pelo método loteria e divididos igualmente entre os GC e GE.

O estudo desenvolvido na China por Liu et al. (2017) contou apenas com participantes idosos ( $n=104)$, distribuídos aleatoriamente em GE $(n=56)$ e GC $(n=48)$. A intervenção analisada pelos pesquisadores compreendeu o uso de uma barreira cutânea moldável desde a formação do estoma, objetivando a redução da incidência de dermatite irritativa, redução no tempo de troca da barreira e maior satisfação no autocuidado dos pacientes.

O ECR de Park, Lee, Oh e Kim (2011) teve como hipótese que uma técnica padronizada em cuidados com estoma (SPSC) resultaria em menor incidência de problemas cutâneos periestomas quando comparada a uma técnica em crosta (TC). Para testar essa hipótese, os pesquisadores selecionaram 81 pacientes atendidos em um hospital terciário da Coreia, que, posteriormente, foram distribuídos nos grupos SPSC $(n=45)$ e TC $(n=36)$ de forma aleatória, por meio de sorteio com moeda (cara e coroa). Ambos os grupos foram instruídos pelas enfermeiras especialistas a higienizar o estoma com água morna e em seguida secar a pele periestoma, além de receberem uma demonstração das técnicas que seriam utilizadas. A TC consistiu na utilização de pó de hidrocoloide e água ou filme líquido de barreira protetora para criar uma membrana artificial, que protege a pele periestoma levemente danificada antes de colocar o equipamento coletor, enquanto a SPSC indica que o sistema seja colado diretamente na pele após os cuidados higiênicos necessários.

Seguimento

O período de follow-up foi diferente nos três estudos, variando de um a três meses de acompanhamento, sendo as avaliações semanais ou mensais. No estudo de Alenezi e Mansour10, o seguimento durou cerca de um mês e meio e as avaliações foram realizadas por meio do instrumento Studio Alterazoni Cutanee Stomale (SACSTM) e da aplicação de um pré e pós-testes para avaliação de satisfação com o autocuidado, com intervalo de uma, três e seis semanas.

Por sua vez, Liu et al. (2017) optaram por uma avalição no momento zero e um mês após a alta hospitalar, por meio de um questionário próprio, o qual investigou a presença ou não de dermatite irritativa e o grau de satisfação. Para classificação da dermatite, os pesquisadores consideraram a presença de vermelhidão, prurido e/ou dor.

Já no estudo de Park et al. (2011), os pesquisadores optaram pela utilização adaptada do Ostomy Skin Tool (OST), levando em consideração a avaliação dos três parâmetros do instrumento: descoloração, erosão e crescimento excessivo do tecido. Os dados foram coletados uma vez por mês após a alta hospitalar, durante três meses consecutivos.

\section{Desfecho}

Alenezi e Mansour (2016) concluíram que a diminuição das complicações na pele periestoma associou-se à educação sobre cuidados com o estoma, haja vista que o estudo apresentou redução significativa dessas complicações no GE. O estudo de Liu et al. (2017) revelou um decréscimo significativo na incidência de dermatite irritativa no GE, bem como um aumento significativo no escore total de satisfação nesse mesmo grupo. Quanto ao tempo na troca dos equipamentos coletores e os custos, não houve diferença significativa entre os grupos, entretanto, o GC utilizou uma maior quantidade de creme barreira 
para evitar vazamento. Por sua vez, Park et al. (2011) observaram que as características de problemas com a pele periestoma analisadas eram mais frequentes no grupo TC, quando comparadas ao grupo SPSC.

\section{Análise do risco de viés}

A avaliação do risco de viés seguiu o método padronizado pela Cochrane e está representada nas Figuras 2 e 3.

Figura 2. Avaliação individual do risco de viés.
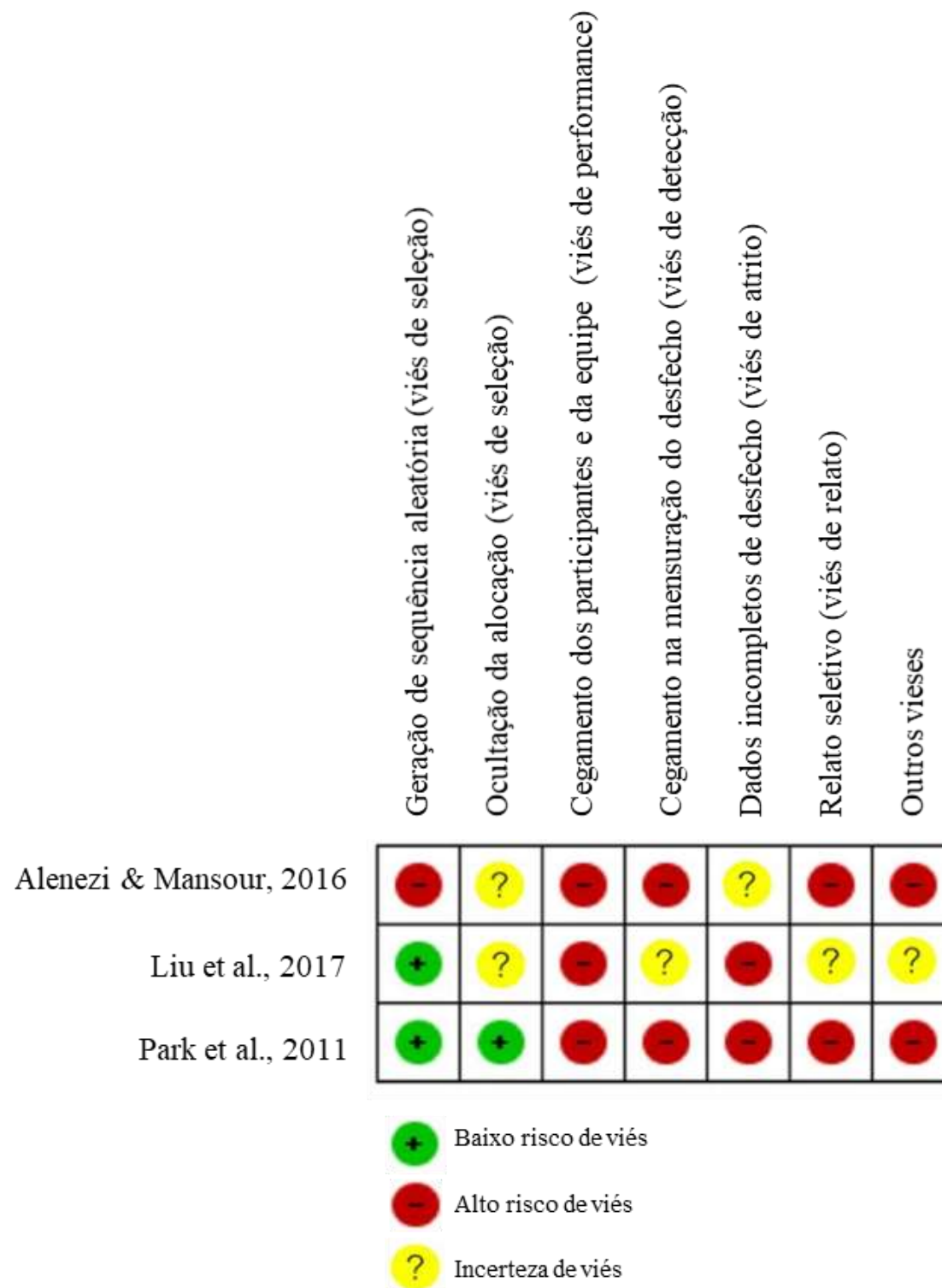

Fonte: Autores. 
Figura 3. Avaliação global do risco de viés.

Geração de sequência aleatória (viés de seleção)

Ocultação da alocação (viés de seleção)

Cegamento dos participantes e da equipe (viés de performance)

Cegamento na mensuração do desfecho (viés de detecção)

Dados incompletos de desfecho (viés de atrito)

Relato seletivo (viés de relato)

Outros vieses
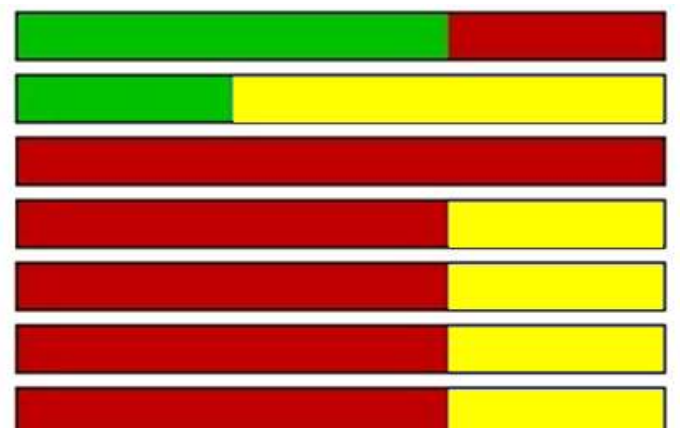

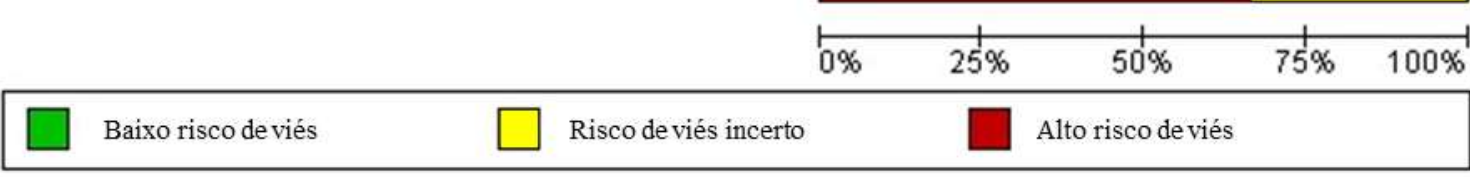

Fonte: Autores.

O trabalho de Liu et al. (2017) foi o estudo que apresentou maior quantidade de itens com baixa clareza metodológica, enquanto o estudo de Park et al. (2011) apresentou a maior quantidade de itens com alto risco de viés (Figura 2). No geral, os estudos apresentaram um alto risco de viés, sendo o mascaramento dos participantes e da equipe o item de maior risco de viés em todos os estudos (Figura 3). Por outro lado, a geração da sequência aleatória de randomização foi analisada como de baixo risco em todos os estudos.

\section{Discussão}

Os resultados dessa revisão sistemática revelam que ainda são escassas evidências científicas sobre as melhores práticas de enfermagem para a prevenção das dermatites periestomas de estomas intestinais. Pesquisas em diferentes cenários são necessárias, pois é sabido que o ambiente e os fatores socioculturais podem influenciar os desfechos relacionados à manutenção da integridade da pele periestoma (Andrade et al., 2017). Entretanto, os ECRs incluídos nesta revisão se mostraram centralizados, tendo em vista que todas as publicações foram realizadas no continente asiático, o que prejudica a validade externa dos estudos e a capacidade de extrapolação dos resultados.

A relação entre a prática de enfermagem e a prevenção de doenças é muito descrita na literatura. O profissional da enfermagem é conhecido como responsável pelo cuidado, pois a promoção e a recuperação da saúde e a prevenção de agravos são pilares do seu exercício profissional (Weber et al., 2019). Contudo, as intervenções em estomaterapia não são simples de serem implementadas, pois o profissional precisa ser técnica e cientificamente habilitado para o manejo de estomas, o que requer um alto investimento (Moraes, Santos, Dantas \& Paula, 2020).

É importante ressaltar que as medidas preventivas propostas no estudo de Alenezi e Mansour (2016) não foram claramente definidas. Os autores não fizeram uma descrição acerca do protocolo, dos métodos e das estratégias utilizadas. Por outro lado, Liu et al. (2017) apresentaram o uso da barreira cutânea moldável como alternativa eficaz ao final de um mês de intervenção, de fácil compreensão e aplicabilidade. Park et al. (2011) sugeriram que o uso ou não do pó de hidrocoloide não interfere diretamente no sucesso do cuidado. Essa abordagem traz consigo algumas violações metodológicas, visto que o desenho do estudo inverte o ônus da prova do conceito.

Em sua pesquisa, Beitz e Colwell (2016) descreveram comentários de enfermeiros especialistas acerca de intervenções para complicações do estoma e de pele periestoma, de acordo com a vivência clínica de cada profissional. Os especialistas sugeriram a identificação e posterior correção da etiologia do contato da pele com o efluente que, por sua vez, 
pode estar relacionada ao equipamento coletor mal ajustado, estoma retraído, hérnia paraestomal, estoma com posicionamento inadequado em dobras na pele, flange reforçada após um vazamento ou deixada na pele por mais tempo que o recomendado pelo fabricante. A preferência pelas barreiras de uso prolongado também pelos autores (Beitz \& Colwell, 2016), uma vez que a frequente troca da barreira adesiva pode levar a lesões na pele periestoma e consequente remoção do estrato córneo. Em casos mais extremos, pode ocorrer a separação da epiderme e derme, o que dificulta a adesão do novo equipamento coletor e aumenta as chances de vazamentos (Almutairi, LeBlanc \& Alavi, 2017).

Embora não haja consenso sobre o tema, alguns autores indicam a troca do equipamento coletor uma vez no período de três a sete dias, de acordo com a durabilidade do produto indicado pelo fabricante (Bafford \& Irani, 2013). É importante ressaltar que essa troca é influenciada por fatores pessoais (preferências), culturais (vestuário e atividades de lazer) e ambientais (temperatura e umidade), porém deve ser sempre planejada (Hey \& Nascimento, 2017).

Atualmente, os pacientes e profissionais de saúde deparam-se com uma variedade de equipamentos coletores e adjuvantes para o cuidado com o estoma e a pele periestoma, desse modo, a sua seleção é de grande importância para a manutenção da integridade da pele. É comum que os pacientes utilizem o sistema convencional de duas peças após a confecção do estoma, o que pode se tornar um desafio para o cliente e/ou seus cuidadores, na medida em que a barreira cutânea convencional deve ser cortada de acordo com o tamanho e formato do estoma, exigindo determinada habilidade técnica. É notório que consequentes complicações podem ocorrer caso o orifício criado não esteja adequado para o tamanho e tipo do estoma (Liu et al., 2017). Por isso, a educação em saúde é fundamental como estratégia acerca dos cuidados com a estomia, com abordagem de acompanhamento contínuo e sistemático visando a garantir o cuidado individualizado (Hoeflok, Guy, Allen \& St-Cyr, 2009).

Salienta-se que a busca pelo gerenciamento de cuidados de pessoas com estomias vem ocorrendo desde séculos passados, em que não havia equipamentos coletores e adjuvantes suficientes e de boa qualidade, o que levava os pacientes a desenvolverem criativamente dispositivos de coleta de efluentes com objetos encontrados no próprio domicílio. Hoje, os achados apoiam e corroboram o trabalho do enfermeiro, principalmente do estomaterapeuta, na prevenção da dermatite periestoma, como uma meta clara para a equipe multidisciplinar, contribuindo e influenciando diretamente na qualidade de vida das pessoas que vivem com uma estomia (Anaraki et al., 2012; Moreira \& Alcântara, 2009).

Todos os estudos incluídos nesta revisão tiveram como amostras pacientes adultos divididos em grupos heterogêneos. Essa estratégia traz consigo a possibilidade de visualização do comportamento em ambos os sexos, todavia faltaram dados para análises comparativas. Levando em consideração o público estudado, vale ressaltar que pesquisas apontam que indivíduos do sexo feminino tendem a se preocupar e dar mais atenção às estratégias de autocuidado (Bussab \& Bolfarine, 2005; Bertolini \& Simonetti, 2014; Moura, Gomes \& Pereira, 2017).

Para avaliar e quantificar as intervenções realizadas nos estudos, foram utilizadas medidas e escalas no processo de avaliação, o instrumento SACSTM, o OST adaptado pelo próprio autor E questionário de mensuração de níveis de satisfação. Sendo este último realizado de forma subjetiva, de acordo com a resposta do paciente, O que torna a estratégia passível de risco. Quando se refere à pesquisa cientifica, os instrumentos e medidas precisam ser bem elencados tendo em vista que a quantificação e qualificação é estratégia fundamental para a obtenção de resultados eficazes, passiveis de serem reproduzidos de maneira confiável (Coluci, Alexandre \& Milani, 2015).

Dois dos três artigos dessa revisão utilizaram instrumentos padronizados e previamente validados na avaliação dos desfechos investigados. O primeiro instrumento, SACSTM, foi utilizado para mensurar a incidência e gravidade das lesões na pele periestoma (Alenezi \& Mansour, 2016). Trata-se de um instrumento desenvolvido e validado na Itália, no qual as lesões são classificadas de acordo com a topografia, dividindo a área periestoma em quatro quadrantes, e pelo tipo de lesão, podendo ser hiperêmico, erosivo, ulcerativo, fibrinonecrótico/necrótico ulcerativo ou proliferativo (Bosio et al., 2007). 
Por sua vez, Park et al. (2011) utilizaram o OST adaptado como instrumento de mensuração do desfecho. Essa ferramenta foi desenvolvida e validada para avaliar as condições da pele periestoma em uma pesquisa envolvendo enfermeiros da Dinamarca e Espanha. O instrumento avalia três itens: descoloração (D), erosão (E) e supercrescimento de tecido (T), sendo que cada item recebe uma pontuação de 0 a 5 , referindo-se à área da lesão (0-3) e gravidade (0-2), totalizando uma pontuação DET de 0 a 15 (Martins et al., 2010). Contudo, Park et al. (2011) não realizaram um processo de validação da escala antes da sua utilização no ECR, o que dificulta a realização de comparação entre estudos que tenham utilizado a mesma ferramenta.

Quanto à eficácia da intervenção aplicada nos grupos estudados, vale ressaltar que o tempo de exposição e acompanhamento são possíveis fatores determinantes. Nos artigos incluídos, não foi possível observada uma padronização, uma vez que o intervalo de follow-up variou de uma a 12 semanas após a alta. Nesse sentido, é necessário que exista uma avaliação crítica da equipe de pesquisadores a respeito do tempo necessário para ocorrência e mensuração do desfecho, o que torna complexos os estudos que envolvem cicatrização e prevenção de lesões. Logo, não é possível inferir a respeito do tempo ideal de acompanhamento para ECRs dessa natureza, baseando-se nos estudos incluídos nessa revisão (Marconi \& Lakatos, 2017).

Os resultados desta revisão sistemática demonstram que a capacitação de enfermeiros deve ser estimulada, sobretudo relacionada às medidas de prevenção de complicações menos onerosas e complexas. No entanto, muitas diretrizes baseiam-se apenas em estudos observacionais, de menor poder de evidência, porque os ECRs nessa área ainda são escassos devido a diversos fatores como baixa adesão dos participantes, indisponibilidade de ambiente adequado para realização da pesquisa, reduzido financiamento (Marcondes et al., 2015).

Os estudos do tipo revisão sistemática de literatura estão no topo da pirâmide das evidências científicas. Um ponto forte deste trabalho é que ele foi desenvolvido seguindo todas as recomendações e diretrizes especializadas. Adicionalmente, trata-se de um estudo inédito e original que contribuirá para a prática de enfermagem baseada em evidências. No entanto, esse estudo tem algumas limitações. Em primeiro lugar, não foi possível realizar a metanálise devido à heterogeneidade dos ECRs incluídos (Pereira, 2010). O escopo da busca esteve voltado para pacientes adultos, desconsiderando as intervenções de enfermagem na neonatologia e pediatria. Além disso, apenas as dermatites periestomas de estomas intestinais foram consideradas e, nesse sentido, o grupo das estomias de eliminação urinárias precisa ser integrado em estudos posteriores. Por fim, o presente estudo avaliou apenas as intervenções de enfermagem. Logo, estudos futuros deverão investigar intervenções multiprofissionais para a prevenção desse tipo de complicação.

\section{Conclusão}

O presente estudo identificou três intervenções de enfermagem utilizadas como medidas de prevenção de dermatite periestoma em estomias intestinais, a saber: a educação sobre os cuidados com as estomias, uso de técnica padronizada para pacientes sem problemas de pele peristoma e aplicação da barreira cutânea moldável. Contudo, não é possível realizar recomendações para a prática devido às limitações do ECRs incluídos.

Embora todos os estudos tenham sugerido que as medidas investigadas são eficazes na prevenção de dermatite periestoma, eles apresentam alto risco de viés e suas pequenas amostras os tornam suscetíveis a erros aleatórios. Portanto, sugerimos que novos ECRs multicêntricos, de alto rigor metodológico e com amostras maiores sejam conduzidos, a fim de proporcionar evidências mais sólidas para a construção de protocolos e diretrizes clínicas de enfermagem em estomaterapia.

\section{Referências}

Alenezi, A. N. \& Mansour, E. A. (2016). Impact of stoma care education in minimizing the incidence of stoma skin complications. Bahrain Medical Bulletin, 38(3), 151-153. 
Almutairi, D., LeBlanc, K., \& Alavi, A. (2017). Peristomal skin complications: what dermatologists need to know. Int J Dermatol, 57(6), 257-264.

Anaraki, F., Vafaie, M., Behboo, R., Maghsoodi, N., Esmaeilpour, S., \& Safaee, A. (2012). Quality of life outcomes in patients living with stoma. Indian J Palliat Care, 18(3), 176-180.

Andrade, R. S., Martins, J. M., Medeiros, L. P., Souza, A. J. G., Torres, G. V., Katherinne, I., et al. (2017). Aspectos sociodemográficos, clínicos e de autocuidado de pessoas com estomas intestinais. Rev Enferm UERJ, 25, e19368.

Bafford, A. C. \& Irani, J. L. (2013). Management and complications of stomas. Surg Clin North Am, 93(1), $145-166$.

Beitz, J. M. \& Colwell, J. (2016). Management approaches to stomal and peristomal complications. J Wound Ostomy Continence Nurs, 43(3), 263-268.

Bertolini, D. N. P. \& Simonetti, J. P. (2014). O gênero masculino e os cuidados de saúde: a experiência de homens de um centro de saúde. Esc Anna Nery Rev Enferm, 18(4), 722-727.

Bosio, G., Pisani, F., Lucibello, L., Fonti, A., Scrocca, A., Morandell, C., et al. (2007). A proposal for classifying peristomal skin disorders: Results of a multicenter observational study. Ostomy Wound Manage, 53(9), 38-43.

Bussab, W. \& Bolfarine, H. (2005). Elementos de amostragem. Edgar Blucher.

Carlsson, E., Fingren, J., Hallén, A. M., Petersen, C., \& Lindholm, E. (2016). The prevalence of ostomy-related complications 1 year after ostomy surgery: a prospective, descriptive, clinical study. Ostomy Wound Manage, 62(10), 34-48.

Carvalho, A. P. V., Silva, V., \& Grande, A. J. (2013). Avaliação do risco de viés de ensaios clínicos randomizados pela ferramenta da colaboração Cochrane. Diagn Tratamento, 18(1), 38-44.

Coluci, M. Z. O., Alexandre, N. M. C., \& Milani, D. (2015). Construção de instrumentos de medida na área da saúde. Ciênc Saúde Colet, 20(3), 925-936.

Gray, M., Black, J. M., Baharestani, M. M., Bliss, D. Z., Colwell, J. C., Goldberg, M. G. et al. (2011). Moisture-associated skin damage: overview and pathophysiology. J Wound Ostomy Continence Nurs, 38(3), 233-241.

Gray, M., Colwell, J. C., Doughty, D., Goldberg, M., Hoeflok, J., Manson, A., et al. (2013). Peristomal moisture-associated skin damage in adults with fecal ostomies: a comprehensive review and consensus. J Wound Ostomy Continence Nurs. 40(4), 389-99.

Hey, A. P. \& Nascimento, L. A. (2017). A pessoa com estomia e o fornecimento de equipamentos coletores e adjuvantes pelo Sistema Único de Saúde. Braz J Enterostomal Ther, 15(2), 92-99.

Hoeflok, J., Guy, D., Allen, S., \& St-Cyr, D. (2009). Prospective multicenter evaluation of a moldable skin barrier of the stoma. Ostomy Wound Manage, $55(5), 62-9$

Hosmer, D. W. \& Lameshow, S. (1989). Applied logistic regression. Wiley.

Hulley, S. B., Cummings, S. R., Browner, W. S., Grady, D. G., \& Newman, T. B. (2013). Designing clinical research. Lippincott Williams \& Wilkins.

Liu, G., Chen, Y., Luo, J., Liu, A., \& Tang, X. (2017). The application of a moldable skin barrier in the self-care of elderly patients with ostomy. Gastroenterol Nurs, 40(2), 117-120.

Loro, M. M., Zeitoune, R. C. G., Guido, L. A., Silveira, C. R., \& Silva, R. M. (2016). Desvelando situações de risco no contexto de trabalho da Enfermagem em serviços de urgência e emergência. Esc Anna Nery Rev Enferm, 20(4), e20160086.

Marcondes, F. L., Tavares, C. M. M., Santos, G. S., Silva, T. N., \& Silveira, P. G. (2015). Capacitação profissional de enfermagem na atenção primária à saúde: revisão integrativa. Revista Pró-UniverSUS, 6(3), 9-15.

Marconi, M. A. \& Lakatos, E. M. (2017). Fundamentos de metodologia científica. Atlas.

Martins, L., Ayello, E. A., Claessens, I., Hansen, A. S., Poulsen, L. H., Sibbald, R. G., et al. (2010). The ostomy skin tool: tracking peristomal skin changes. Br J Nurs, 19(15), 932-934.

Moher, D., Liberati, A., Tetzlaff, J., \& Altman, D. G. (2009). Preferred Reporting Items for Systematic Reviews and Meta-Analyses: The PRISMA Statement. PLoS Med, 6(7), e1000097.

Moraes, J. T., Santos, V. L. C. G., Dantas, S. R. P. E., \& Paula, M. A. B. (2020). Cursos de estomaterapia acreditados para uma formação de excelência. Braz J Enterostomal Ther, 18, e0420.

Moreira, T. M. M. \& Alcântara, M. C. M. (2009). Enfermagem em estomaterapia: cuidados clínicos ao portador de úlcera venosa. Rev Bras Enferm, 62(6), $889-893$.

Moura, E. C., Gomes, R., \& Pereira, G. M. C. (2017). Percepções sobre a saúde dos homens numa perspectiva relacional de gênero, Brasil, 2014. Ciênc Saúde Coletiva, 22(1), 291-300.

Nadal, R. V., Peñalba, A. M. V., \& Zamora, M. E. I. (2007). Dermatosis alrededor de ostomías. Piel, 22(3), $119-31$.

Park, S., Lee, J. Y., Oh, D. N., \& Kim, J. (2011). Comparison of standardized peristomal skin care and crusting technique in prevention of peristomal skin problems in ostomy patients. J Korean Acad Nurs, 41(6), 814-820.

Pereira, M. M. B. (2010). Sobre a revisão sistemática e a meta-análise na área da fluência. Rev CEFAC, 12(1). 
Research, Society and Development, v. 10, n. 7, e48110716740, 2021

(CC BY 4.0) | ISSN 2525-3409 | DOI: http://dx.doi.org/10.33448/rsd-v10i7.16740

Salvadalena, G. Incidence of complications of the stoma and peristomal skin among individuals with colostomy, ileostomy, and urostomy: a systematic review. (2008). J Wound Ostomy Continence Nurs, 35(6), 596-607.

Shea, B. J., Grimshaw, J. M., Wells, G. A., Boers, M., Andersson, N., Hamel, C. et al. (2007). Development of AMSTAR: a measurement tool to assess the methodological quality of systematic reviews. BMC Medical Research Methodology, 7, 10.

Silva, J. O., Gomes, P., Gonçalves, D., Viana, C., Nogueira, F., Goulart, A. et al. (2019). Qualidade de vida nos doentes ostomizados - estudo usando o questionário Stoma-care QoL - influência de alguns dados clínicos e demográficos na QoL. J Coloproctol, 39(1), 48-55.

Weber, M. L., Vendruscolo, C., Adamy, E. K., Lorenzon, T. L. N., Ferraz, L., \& Zamatta, E. A. (2019). Prática de enfermagem baseada em evidências e suas implicações no cuidado: uma revisão integrativa. Revista Enferm Atual In Derme, 90(28), 1-8.

Wick, E. C., Shore, A. D., Hirose, K., Ibrahim, A. M., Gearhart, S. L., Efron, J. et al. (2011). Readmission rates and cost following colorectal surgery. Dis Colon Rectum, 54(12), 1475-1479. 